\title{
Nanotheranostics
}

2020; 4(4): 201-209. doi: 10.7150/ntno.41639

Research Pape

\section{A Gold Nanoparticle Bouquet held on plasma membrane: An ultrasensitive dark-field imaging approach for Cancer Cell Analysis}

\author{
Yue Cao ${ }^{\bowtie}$, Jie Wang, Qiao-Yan Jiang, Li Hu, You-Jia Yu, Yan-Fang Yu, Feng Chen ${ }^{\bowtie}$ \\ Department of Forensic Medicine, Nanjing Medical University, Nanjing, Jiangsu, 211166, PR China. \\ $\triangle$ Corresponding author: E-mails: ycao@njmu.edu.cn (Yue Cao); E-mail: fchen@njmu.edu.cn (Feng Chen). \\ (C) The author(s). This is an open access article distributed under the terms of the Creative Commons Attribution License (https://creativecommons.org/licenses/by/4.0/). \\ See http://ivyspring.com/terms for full terms and conditions.
}

Received: 2019.11.12; Accepted: 2020.06.08; Published: 2020.06.20

\begin{abstract}
Rational: $\mathrm{p} 53$ is suppressing tumor protein correlated with the cell cycle factors and apoptosis. Here, a gold nanoparticle bouquet is designed for an ultrasensitive dark-field imaging approach for cancer cell analysis.

Methods: AuNP60/APBA is functionalized by a gold nanoparticle bouquet-plasmonic $60 \mathrm{~nm}$ gold nanoparticles. And consistent APBA can be held on the plasma membrane. After $13 \mathrm{~nm}$ gold nanoparticles are functionalized with mannose (AuNP13/MN), the AuNP60/APBA gold nanoparticles are captured. The absorption spectrum of aggregation gold nanoparticles (AuNPs) shifts to near-infrared (NIR) region which can be observed under dark-field microscopy (DFM) and is treated the subsequent with photothermal therapy.

Results: The results that MCF-7 cells were successfully destroyed under the near-infrared (NIR) irradiation and the intracellular WTp53 increased while the MTp53 decreased. These results indicated that $\mathrm{p} 53$ is the key molecule in the apoptosis signaling pathway. Photothermal therapy can stimulate the MTp53 in the cell signal conductive pathway.

Conclusion: This work offers a new method for intracellular p53 analysis and a potential targeted cancer treatment.
\end{abstract}

Key words: gold nanoparticle bouquet, dark-field imaging, p53, cancer cell, photothermal therapy

\section{Introduction}

Noble metal nanoparticles, peculiarly gold nanoparticles (AuNPs), keep attracting much attention due to its excellent biocompatibility and picturesque optical properties [1-5]. AuNP is an attractive nanomaterial candidate with resonant absorption and excitation of incident light to induce strong local surface plasmon resonance (LSPR) [6,7]. With the continuous development of dark-field hyperspectral imaging (DFM) and plasmon resonance scattering spectrum, the impact of plasmonic nanoparticles has been successfully applied in biosensing [6], single particle analysis [8], colorimetric biosensors [9], and single-cell monitoring [10]. On one hand, AuNPs has a strong absorption capacity and high heat conversion efficiency, which was also inspired by the AuNPs' plasma properties and excellent tolerability [11]. Although AuNP-based light therapies for cancer have been widely reported [12-14], these methods lack the ability to do cell surface photothermal therapy or track cancer marker p53. In this field, it's worth emphasizing that how flexibility to design new-style AuNP-based photothermal reagents for high sensitivity and selectivity of cancer therapy. On the other hand, taken the clinical trials consideration the clinical trials, it is expected that the photothermal emission of 
absorption of long-wave light in the near-infrared (NIR) region can further penetrate the tissue $[15,16]$. To accomplish this target, AuNPs of varying forms and sizes have been prepared for NIR illumination and attained a good therapeutic effect [17]. Regrettably, these nanoparticles cannot fixedly aggregate on the cell membrane since they can easily be endocytosed into cells. This prompted us to design an intelligent system that assembled on the surface of cancer cells after the small-sized AuNPs (The diameter of which is less than $20 \mathrm{~nm}$ ) capture the 60 $\mathrm{nm}$ gold which is immobilized on the cell membrane. The system can form a gold nanobeam, whose absorbing light is in the near-infrared region, for subsequent photothermal therapy.

Human p53 is a tumor-suppressing protein to controls cell dividing cycle and apoptosis (programmed cell death) by controlling tumor progression [18]. There is a central specific DNAbinding domain in present human WTp53 (wild-type p53 protein) [19]. The DNA-binding center region of p53 protein loses binding capacity due to extensive mutation in p53 gene in various types of cancer cells [20]. Abnormal expression of MTp53 (mutant-type p53 protein) can serve as an important carcinogenesis stimulus [21]. Thus far, various methods for detecting endogenous p53 have been reported, including enzyme-linked immunosorbent assay (ELISA) [22], immunohistochemically assay [23], electrophoretic mobility shift assay [24], and ds-DNA consensus binding biosensing assay [25]. Despite the favorable detection limit and sensitivity of these methods, none of these methods linked at the relationship between photothermal therapy and p53 protein signaling pathways. Hence, it is urgently needed to design in situ analysis strategies for photothermal therapy of cell membrane surface to analyzing the changes of p53 protein in cells.

To investigate whether the gold nanoparticle bouquet can downregulate MTp53 during photothermal therapy, the gold nanoparticle bouquet (AuNP60/APBA-AuNP13/MN) was designed through dual functionalization of a single 3aminophenyl boronic acid (APBA) assembled on the surface of gold nanoparticles (AuNP60) with aminegold links to construct AuNP60/APBA. APBA binds to mannan-conjugated gold nanoparticles (AuNP13/ $\mathrm{MN})$ [26,27]. The AuNP60/APBA, which is a plasmonic AuNP, can held on the plasma membrane through the competitive reaction of cell surface SA with AuNP13/MN. Thus SA can replace AuNP13/ $\mathrm{MN}$ and bound to AuNP60/APBA. AuNPs absorption can be transferred to near-infrared by polymerization. They can not only can be viewed under dark-field hyperspectral imaging (DFM), but also be used for the subsequent photothermal therapy. It is showed that MCF-7 cells were successfully broke under-near-infrared (NIR) irradiation. And the intracellular WTp53 increased while the MTp53 decreased (Figure 1). These results indicated that p53 is the key molecule in the apoptosis signaling pathway. Photothermal therapy can stimulate the MTp53 in the cell signal conductive pathway. This research affords a new strategy for studying intracellular p53 analysis through photothermal therapy on the cell surface and finds a potential in-situ treatment against cancer.

\section{Experimental Section}

\section{Sample Preparation for the Detection with Dark-Field Microscopy (DFM) and Scattering Spectroscopy}

The AuNPs that were used for DFM images were fixed on the surface of the glass sheet. The glass sheet was sonicated in ethanol and then washed with pure water. The clean glass sheet was dried, and the AuNP solution was deposited on the surface to evaluate the DFM detection. To image cells that had absorbed AuNPs, the cell suspension $\left(1 \mathrm{~mL}, 1 \times 10^{6} \mathrm{~mL}^{-1}\right)$ was seeded in each culture dish (the bottom is glass sheet) and cultured overnight. Then, $30 \mu \mathrm{L}$ of the AuNP-60/APBA was added to each dish and incubated for various times at $37^{\circ} \mathrm{C}$. Furthermore, the dishes were washed twice and immersed in PBS for DFM imaging. Then, $50 \mu \mathrm{L}$ of the AuNP-wrapped mannan was added to each dish. The AuNP-scattered light was split using a grating, and the scattering spectra of the samples were recorded by CCD spectrometer.

Supporting Information (Experimental Section).

\section{Result and Discussion}

\section{Characterization of the gold nanoparticle bouquet}

The preparation of $60 \mathrm{~nm}$ AuNPs is by the method previously reported [28]. The AuNPs were modified APBA and could identify the sialic acid SA site. The mannan-conjugated $13 \mathrm{~nm}$ AuNPs were synthesized through a one-pot synthetic process with mannose-polysaccharide mannan as stabilizer and $\mathrm{NaBH} 4$ as the reducing reagent.[29]. The image of AuNP60/APBA was taken by transmission electron microscopy (TEM). We can see from Figure 2A-1 that the average size of the AuNP60/APBA nanoparticles is $60 \mathrm{~nm}$ and it distributes evenly. Figure 2A-2 indicates that the diameter of the AuNP13/MN diameter is $18 \mathrm{~nm}$, which is consistent with the 
dynamic light scattering (DLS) result of $18 \mathrm{~nm}$ and is 5 nm larger than the naked AuNPs synthesized under the same condition without the presence of mannan (Figure 2C). TEM images of the AuNP60/APBA incubated with AuNP13/MN at different concentrations were shown in Figure 2 A-3, Figure 2B, B-1， B-2, B-3， B-4, B-5. The AuNP13/MN, AuNP60/APBA and AuNP60/APBA-AuNP13/MN nanoparticles presented a negative zeta potential (Figure S1). As shown in Supporting Information Figure S2, green spots (AuNPs) were observed from the DFM image. The modified AuNP60/APBA still has green spots, which means that the diameters of the AuNP60/APBA and the bald AuNPs are the same. The UV-Vis spectrum of the AuNP60/APBA showed a characteristic peak at $535 \mathrm{~nm}$, which red shifted to $548 \mathrm{~nm}$ after added AuNP13/MN (522 nm) was conjugated on their surface, which demonstrated the successful connection of the AuNP13/MN and
AuNP60/APBA (Figure 2D). The average amount of APBA assembled on each nanoparticles was measured to be around $1.44 \times 10^{6}$. The concentrationdependent temperature increase of AuNP60/APBAAuNP13/MN nanoparticles bouquent was detected with the extension of laser illumination time, as indicated in Figure S3A. After $50 \mathrm{~min}$ of irradiation, the temperature of AuNP60/APBA-AuNP13/MN aqueous solution was increased to $42^{\circ} \mathrm{C}$ at the maximum concentration of $10 \mathrm{~nm}$ (Figure S3B). Then, the photothermal conversion efficiency ( $\eta$ ) of AuNP60/APBA-AuNP13/MN nanoparticles bouquent exposed to $680 \mathrm{~nm}$ lasers for $500 \mathrm{~s}$ was performed and the photothermal conversion efficiency value of AuNP60/APBA-AuNP13/MN nanoparticles bouquent was calculated to be $40.6 \%$. [30] (The detailed process is provided in the Supporting Information.

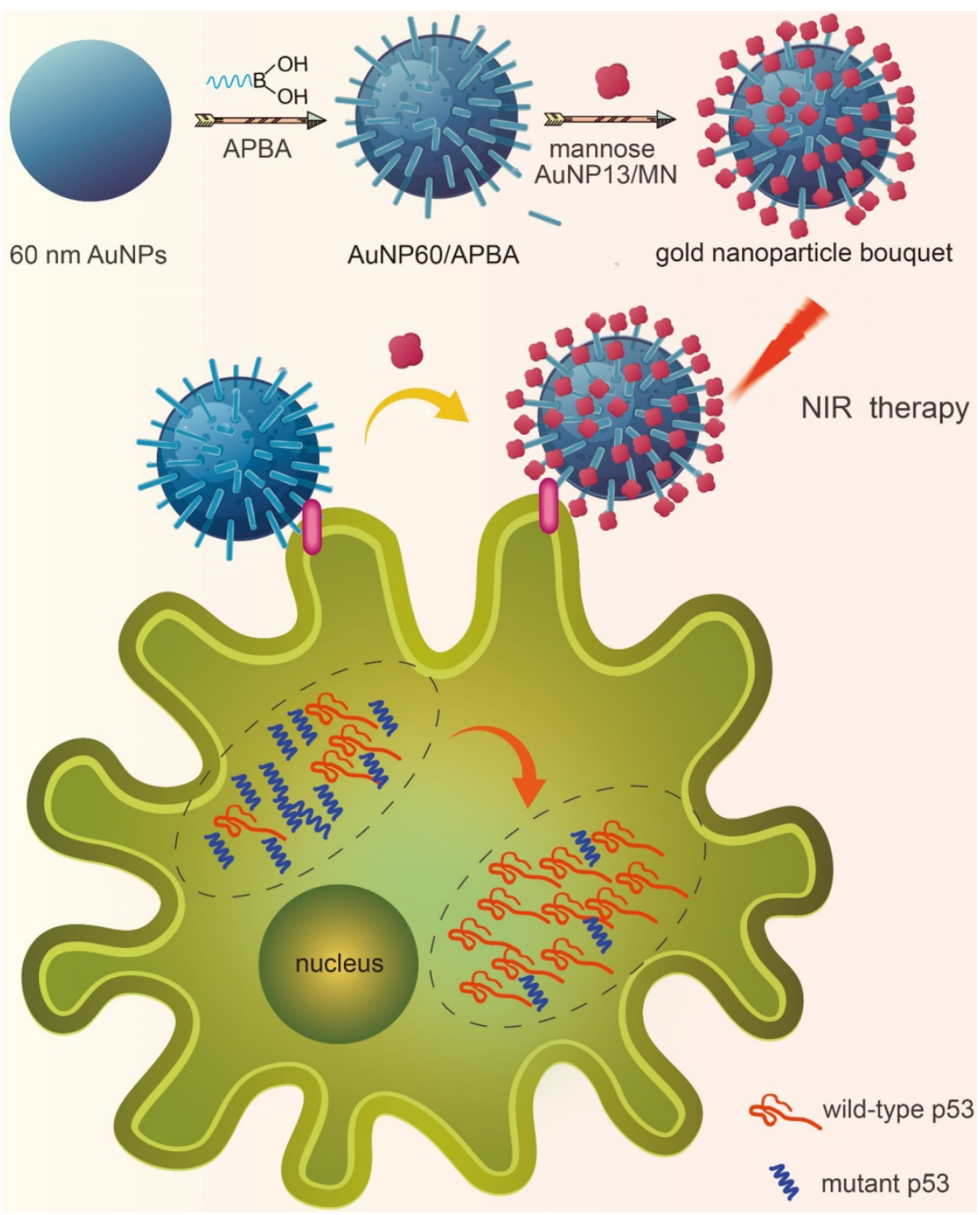

Figure 1. A schematic illustration of a gold nanoparticle bouquet (AuNP60/APBA-AuNP13/MN) for in situ analysis of in-tracellular WTp53 and MTp53. 
A

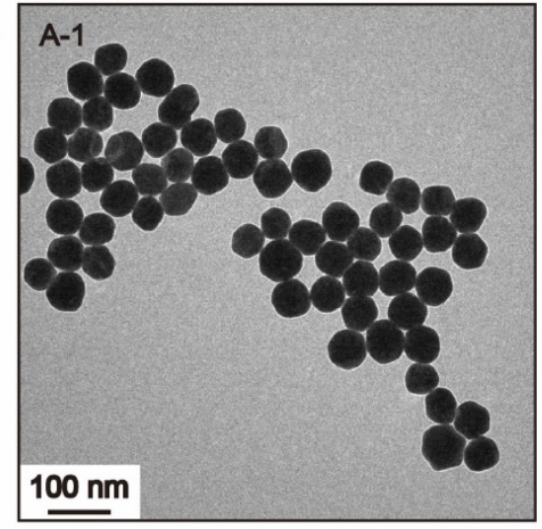

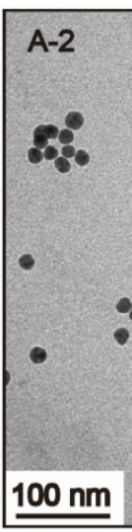
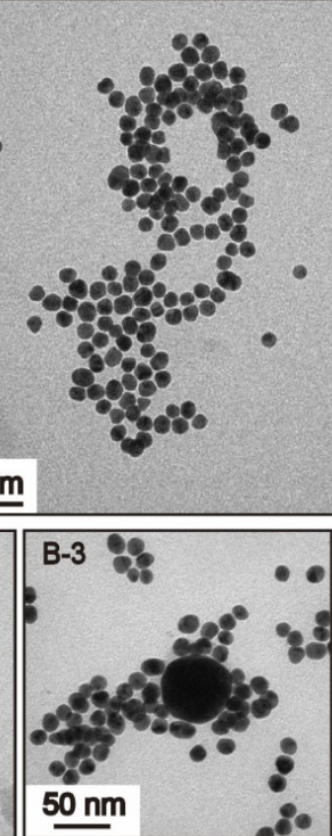

$50 \mathrm{~nm}$

C
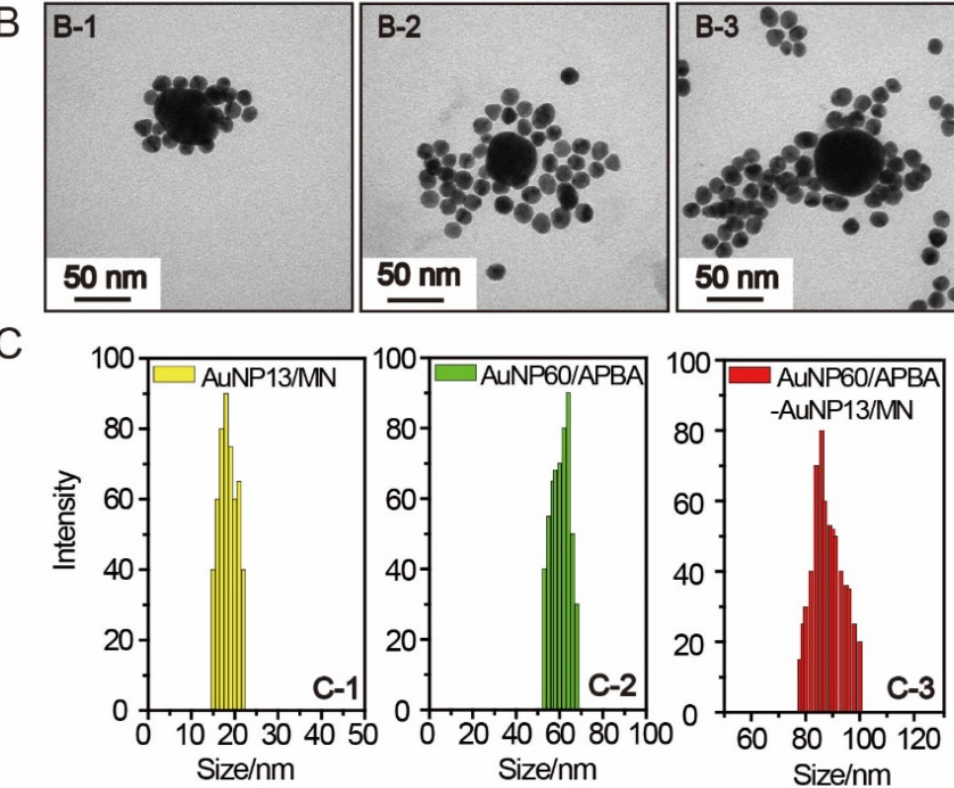
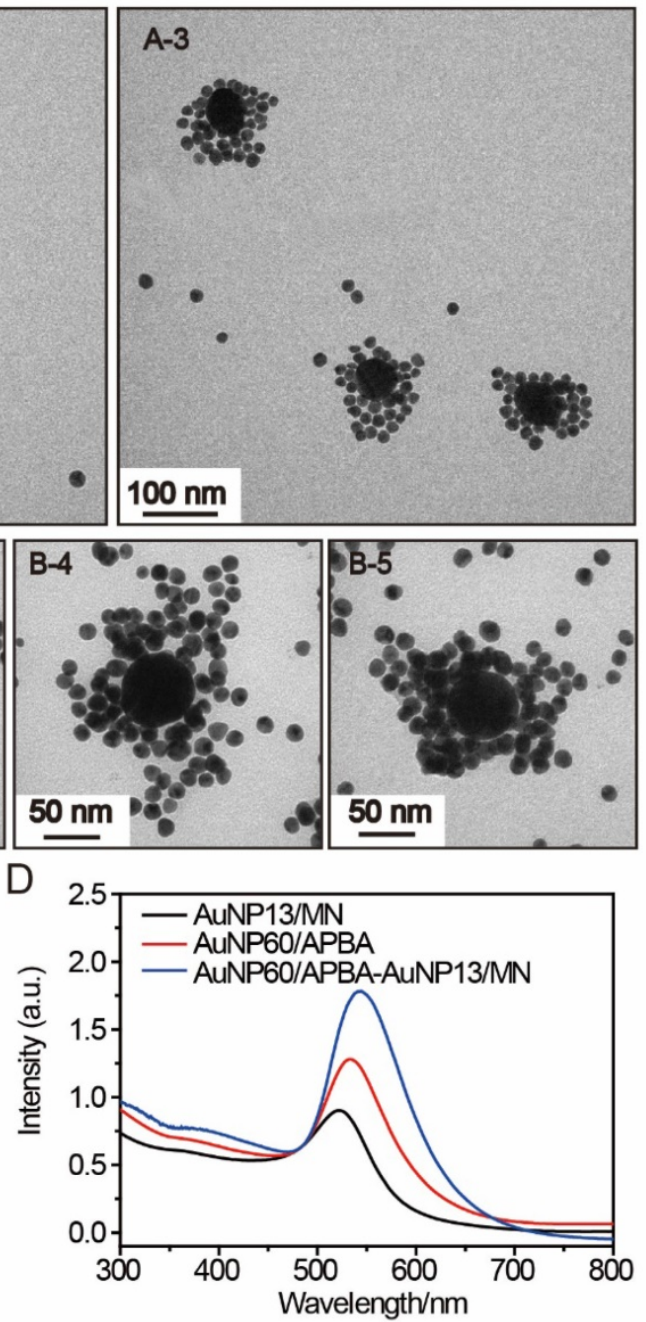

Figure 2. (A) TEM image of the prepared AuNP60/APBA (A-1), AuNP13/MN (A-2) and AuNP60/APBA-AuNP13/MN (A-3). (B) TEM images of the AuNP60/APBA incubated with AuNP13/MN at different concentration (final AuNP13/MN concentration: 2, 4, 6, 8 and $10 \mathrm{nM}$ from (B-1) to (B-5). (C) Dynamic light scattering (DLS) result of the prepared AuNP13/MN, AuNP60/APBA and AuNP60/APBA-AuNP13/MN. (D) UV-Vis spectra of AuNP13/MN, AuNP60/APBA and AuNP60/APBA-AuNP13/MN.

\section{In situ Dark Imaging of the gold nanoparticle bouquet}

To optimize the incubation time for the binding reaction between AuNP60/APBA and AuNP13/MN in solution, the DFM images were recorded at different time points after a series of AuNP13/MN solution $(50 \mu \mathrm{L})$ were incubated with AuNP60/APBA $(30 \mu \mathrm{L}, 5 \mu \mathrm{M})$ at $37^{\circ} \mathrm{C}$. As shown in Supporting Information Figure S4, the color of AuNPs by degrees changed from green to orange and stopped changing after $100 \mathrm{~min}$, which demonstrated the aggregation of AuNP13/MN and AuNP60/APBA-triggered. A series of different concentrations of AuNP13/MN solutions were incubated with AuNP60/APBA (30 $\mu \mathrm{L}, 5 \mu \mathrm{M})$ solution according to the optimum reaction time. Figure $3 \mathrm{~A}$ demonstrated this by the fact that the color was green at a low concentration and the peak of the scattering spectrum was basically the same as that of the control group (Figure 3Aa, g1). The interaction between the AuNP60/APBA and the increase of AuNP13/MN concentration caused a dramatic color change to yellow and then orange and the scattering peak shifted from $550 \mathrm{~nm}$ to $610 \mathrm{~nm}$ upon the AuNP13/MN and AuNP60/APBA conjugation (Figure 3A b-f, g2-g6). Therefore, we developed an expedient quantification approach for AuNP60/ APBA DFM images processed with AuNP13/MN at different concentrations. Corresponding statistical scattering spectral peak of the AuNP60/APBA was determined using the Gaussian function (Figure 3B). The distribution histogram showed the relationship between the AuNP60/APBA and different concentrations of the AuNP13/MN.

\section{In vitro Studies}

MCF-7 cells $\left(1 \mathrm{~mL}, 1 \times 10^{6} \mathrm{~mol} \cdot \mathrm{L}^{-1}\right)$ were planted in a petri dish for intracellular testing. After the 
adding of $30 \mu \mathrm{L}$ AuNP60/APBA, DFM images were obtained at different time points. Only a small amount of AuNP60/APBA particles were observed in the first $30 \mathrm{~min}$. After $1 \mathrm{~h}$, significantly more AuNP60/APBA nanoparticles appeared on cell membrane surface. The number of AuNP60/APBA increased with the extended incubation time until reached the plateau after 2 h (Supporting Information, Figure S5). MCF-7 cells incubated with different concentrations of AuNP13/MN were analyzed by DFM imaging and scattering spectroscopy at $2 \mathrm{~h}$ optimum incubation time (Figure 4). From Figure 4 A1-A3, the AuNP60/ APBA nanoparticles bouquet had a green color and has a peak scattering spectrum at approximately 520 nm (Figure 4, A4-A6), which indicated no AuNP13/ $\mathrm{MN}$ in the MCF-7 cells. However, with increasing doses of AuNP13/MN, the color of AuNP60/APBA nanoparticle bouquet became orange in the DFM images (Figure 4, B3, C3, D3), and the scattering peak gradually redshifted from $520 \mathrm{~nm}$ to $610 \mathrm{~nm}$ little by little (Figure 4, B4-B6; C4-C6; D4-D6), after incubation with different concentration of the AuNP13/MN for 2 $\mathrm{h}$, these changes indicated that the formation of AuNP60/APBA nanoparticle bouquet depends on the dose-dependent increase of AuNP13/MN in intracellular.

In addition, after the intracellular DFM images, the feasibility of p53 apoptotic signalling pathway based on the AuNP60/APBA nanoparticle bouquet was applied by MCF-7 cell-free extracts. The change of p53 level in MCF-7 cells was studied by using AuNP60/APBA nanoparticle bouquet as a simulation drug through NIR irradiation. After incubated with AuNP60/APBA for $2 \mathrm{~h}$, the MCF-7 cells $\left(1 \mathrm{~mL}, 1 \times 10^{6}\right.$ $\mathrm{mL}^{-1)}$ extracts were added with different amounts of AuNP13/MN solution for 12h. A p53 pan ELISA kit (KeyGen Biotech. Co. Ltd., Nanjing, China) was used to detect the level of total p53 (WTp53 and MTp53), extraction of the above cell extracts for testing and establishment of the standard calibration curve (Supporting Information, Figure S6, Figure S7). After drug treatment, the total p53 level was increased, while the MTp53 level gradually declined (Figure 5A), which indicated through NIR irradiation of AuNP60/APBA-AuNP13/MN nanoparticle bouquet ability to stimulate the MTp53 in cell signal conductive pathway.

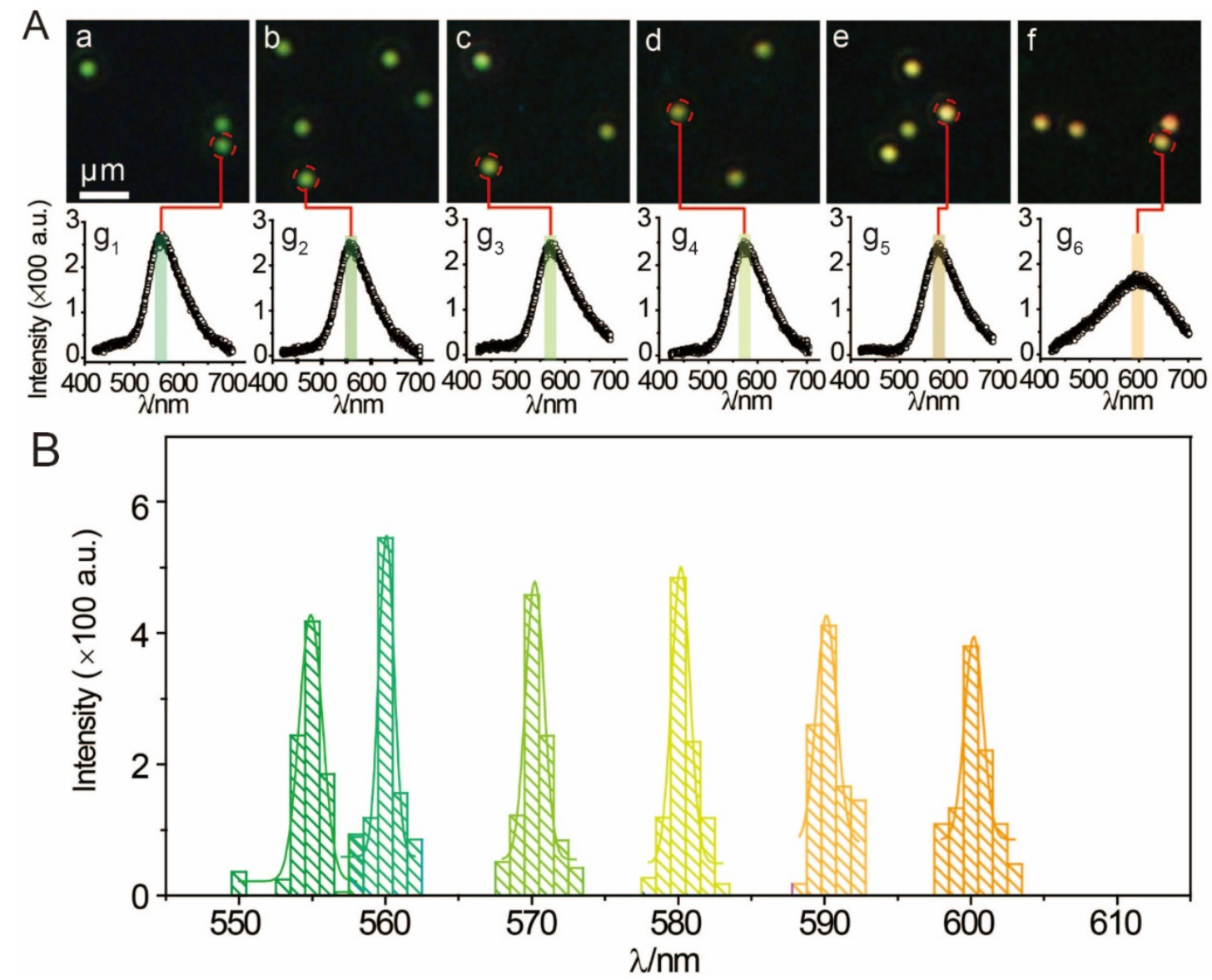

Figure 3. (A) DFM images of $30 \mu \mathrm{L}$ AuNP60/APBA incubated with $50 \mu \mathrm{L}$ AuNP13/MN for 100 min with different concentrations (final AuNP13/MN concentration: $0,2,4,6,8$ and $10 \mathrm{nM}$ from (a) to (f). g1)-g6): Corresponding scattering spectra of AuNP60/APBA observed in DFM images. (B) Corresponding statistical graph of AuNP60/APBA in DFM images a-f in (A). 


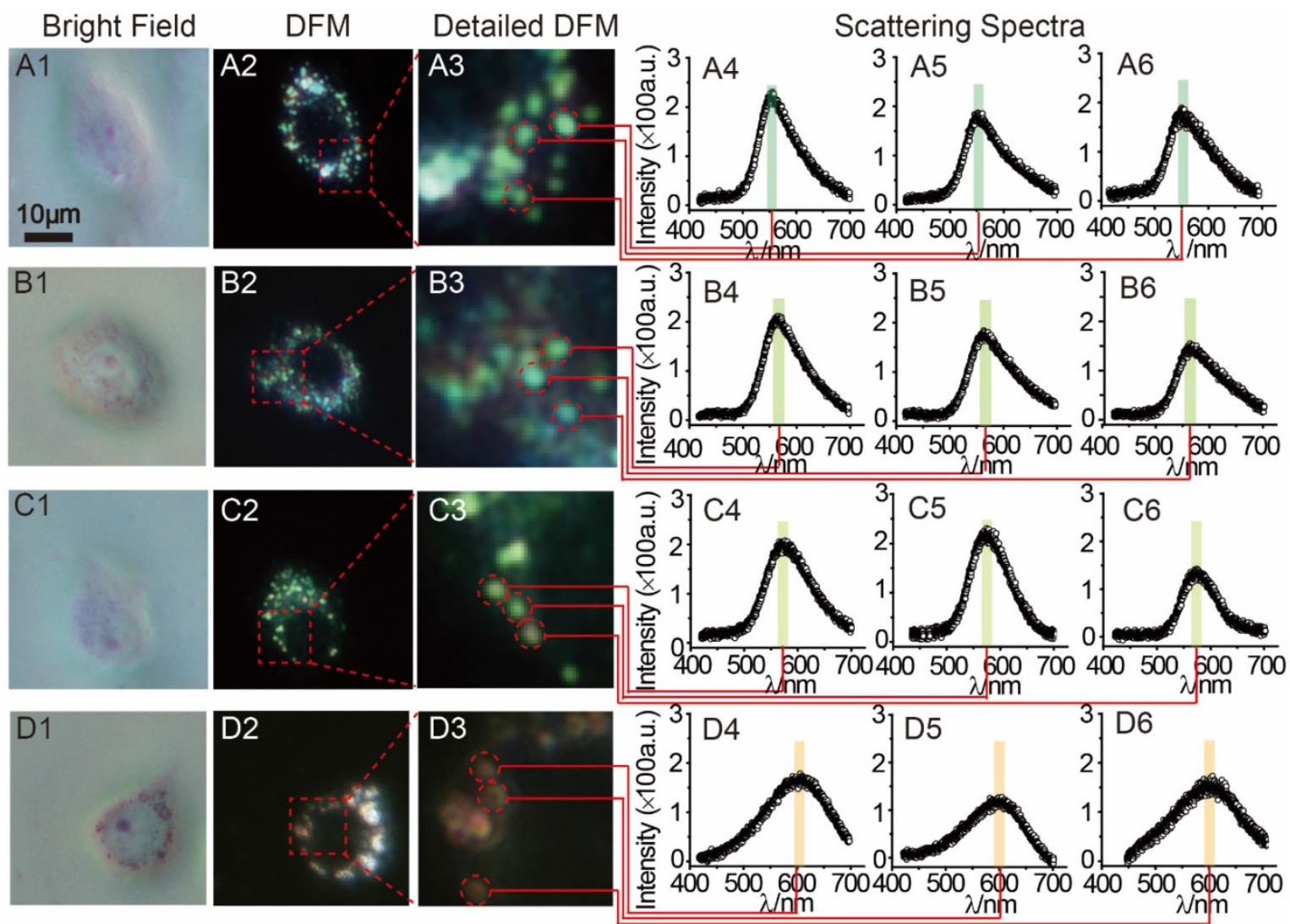

Figure 4. DFM images of $30 \mu \mathrm{L}$ AuNP60/APBA incubated with a single MCF-7 cell (treated with $0,2,5$ and $10 \mathrm{nM}$ ) AuNP13/MN from A to D) for 100 min. (Al-D1: bright field; A2-D2: DFM images; A3-D3: Detailed DFM; A4-6-D4-6: corresponding scattering spectra).

To demonstrate that photothermal therapy using AuNP60/APBA-AuNP13/MN nanoparticle bouquet could adjust MTp53 content, WTp53 and MTp53 in the MCF-7 cell extracts were collected respectively for measurement after the therapy. The result showed that the expression of WTp53 was upregulated while the expression of MTp53 was downregulated. The identification of WTp53 and MTp53 in HBE cells, a non-tumor cell line, was used as control-experiment (Figure 5B). P53 levels were normal in the HBE cells, but MTp53 levels were abnormal in MCF-7 cells. At the same time, when the AuNP60/APBA-AuNP13/ MN nanoparticle bouquet was added to MCF-7 cells for photothermal treatment, the MTp53 levels have been decreased by a half and the number of WTp53 increased by one times (Figure 5B). The results indicate that photothermal therapy can stimulate the MTp53 in the cell signal conductive pathway and the cells enter normal apoptosis state.

The above results have successfully proved that the proposed binary system can efficiently bind AuNP probe on the surface of living cells to form a polymer in the presence of AuNP13/MN. MCF-7 cells were used as a model to investigate whether the aggregates could be applied to kill tumor cells under NIR photothermal therapy. After incubation with the different concentrations of AuNP60/APBA nanoparticle bouquet for $12 \mathrm{~h}$, the cells were irradiated with a laser $\left(680 \mathrm{~nm}, 0.5 \mathrm{~W} \mathrm{~cm}^{-2}, 37^{\circ} \mathrm{C}\right)$. The absorbances were examined to ascertain the relative cell activity. Cell viability remained at $90 \%$ in the dark (Supporting Information, Figure S8), suggesting that AuNP60/APBA nanoparticle bouquet were biocompatible in cell experiments. However, after 20 min of NIR irradiation, morphological changes of cells (Figure S9) and the apoptotic MCF-7 cells bursted, which demonstrated that AuNP60/APBA nanoparticle bundles were capable of killing cancer cells under NIR illumination in a cell biocompatibility assay system (Figure S8). Meanwhile, consistent with the results from the MTT assay, EdU incorporation assay (Figure 5C and 5D) and TUNEL assay (Figure $5 \mathrm{E}$ and $5 \mathrm{~F}$ ) revealed that the proliferation of MCF-7 cells was reduced while the apoptosis was increased in light group. TEM images showed the AuNP60/ APBA-AuNP13/MN nanoparticle bouquet in MCF-7 cell surface (Figure S10). Overall, these results revealed that AuNP60/APBA-AuNP13/MN nanoparticle bouquet combined with illumination showing an excellent anti-tumor effect on human MCF cells. 

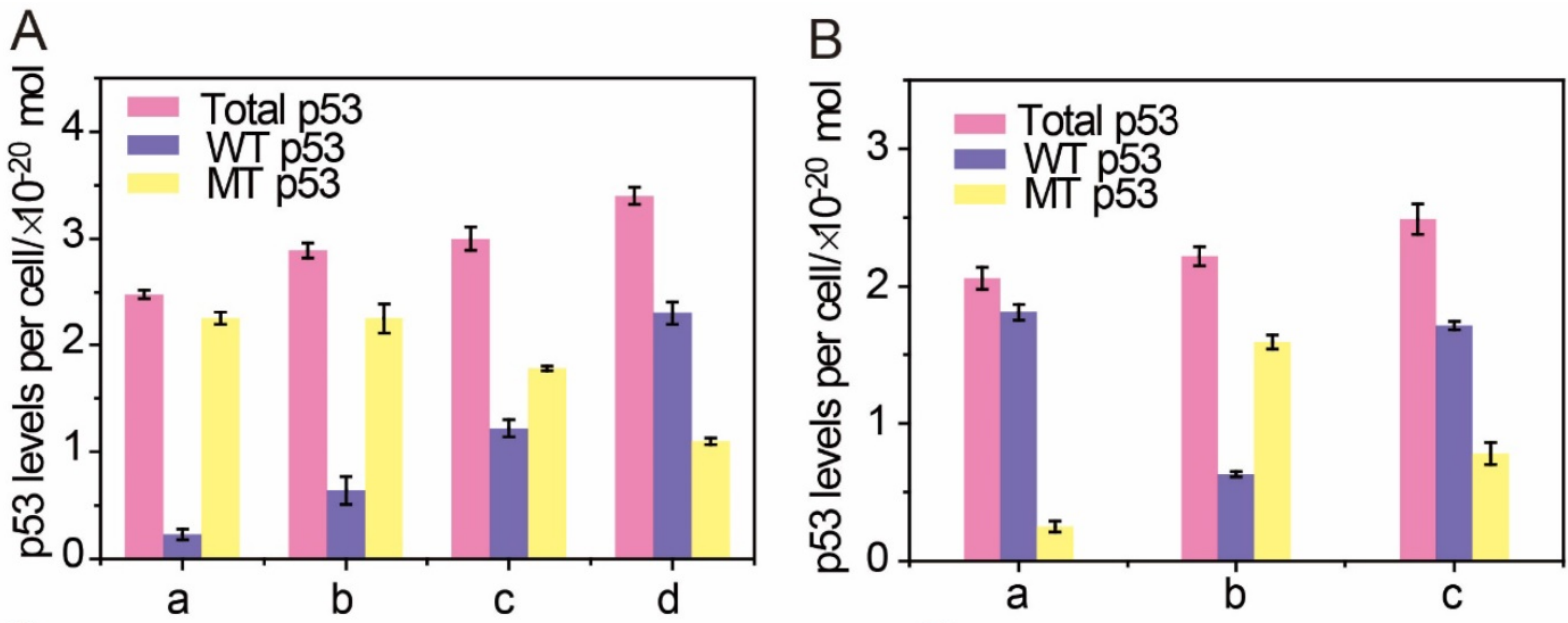

C
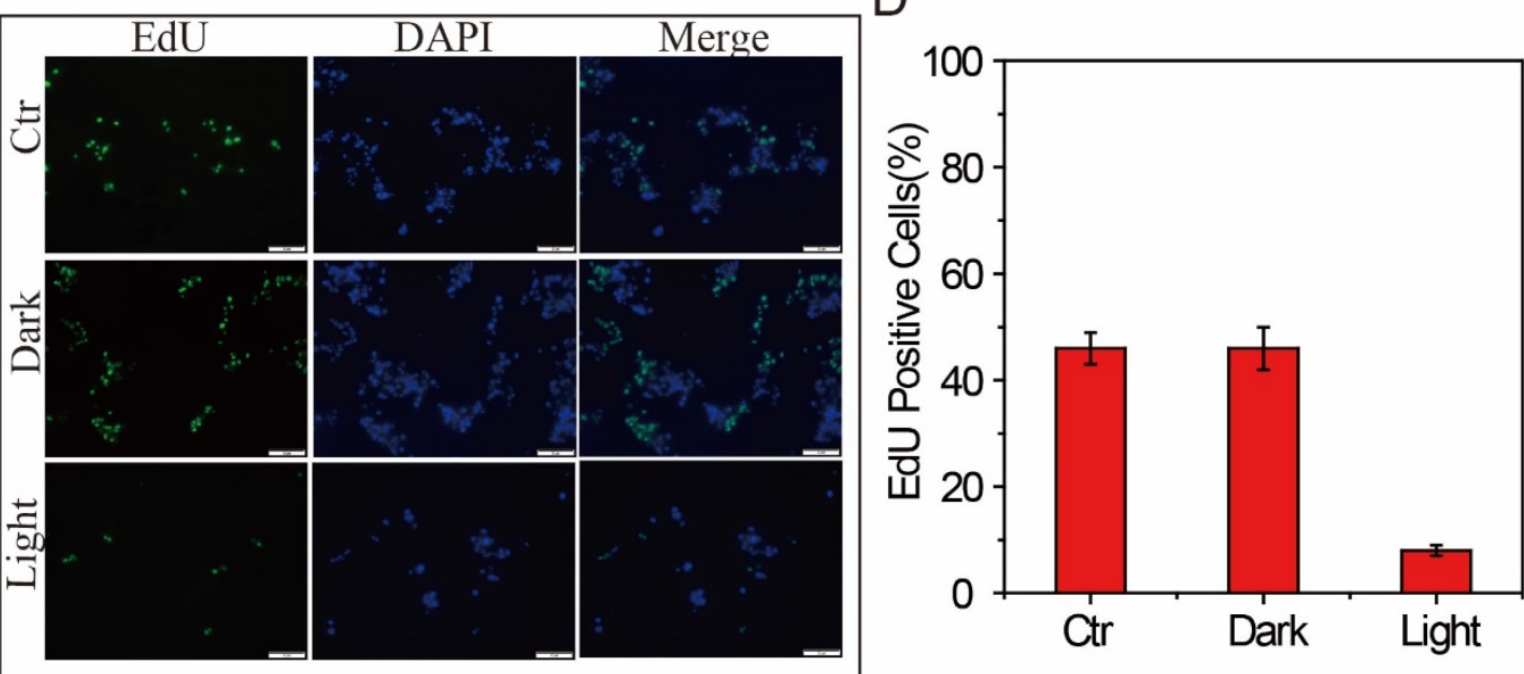

E

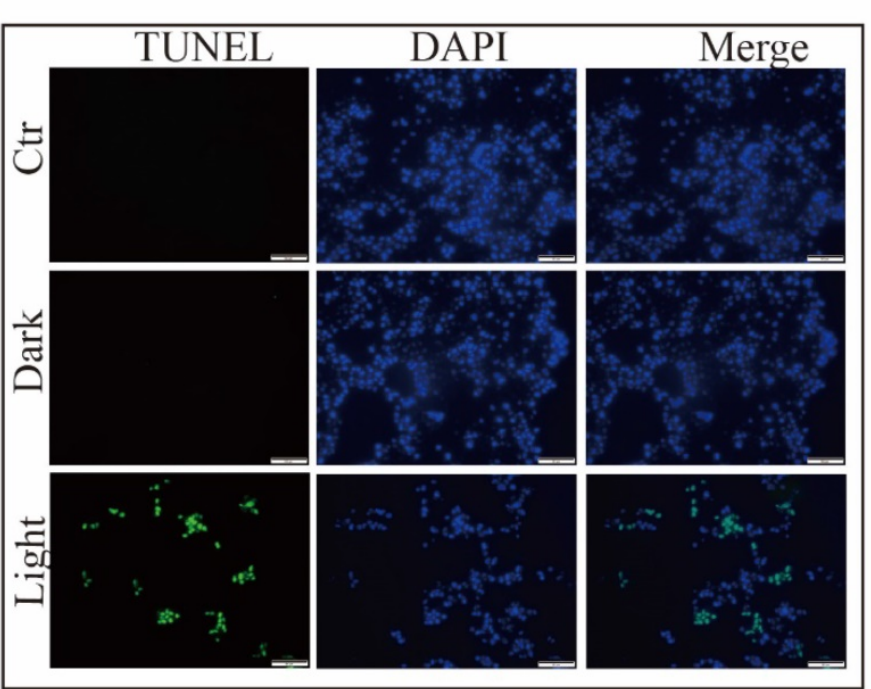

$\mathrm{F}$

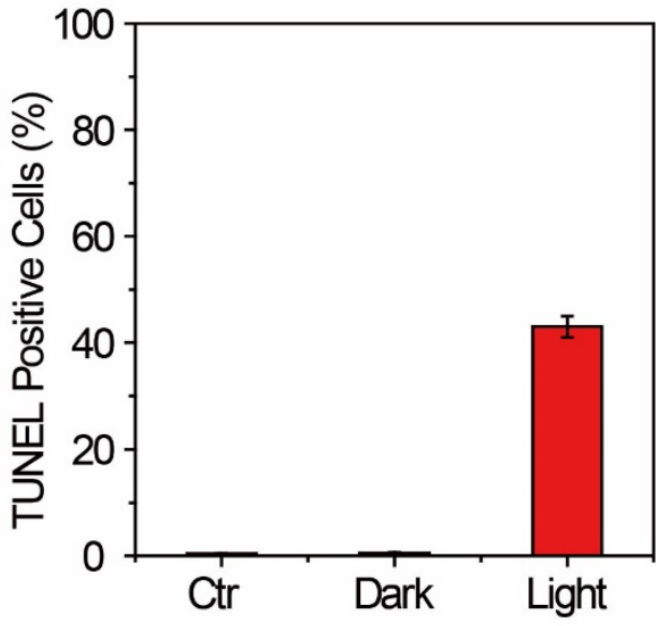

Figure 5. (A) p53 levels (total p53, WTp53 and MTp53) in MCF-7 cell (detected from cell extracts collected from MCF-7 cells treated with: AuNP60/APBA contain (no, 2, 5, $10 \mathrm{~nm}$ ) AuNP13/MN from A to D. (B) p53 levels (total p53, WTp53 and MTp53) in (A: HBE cells; B: MCF-7 cells; C: Added AuNP60/APBA-AuNP13/MN nanoparticle bouquet to MCF-7 cells. (C) EdU incorporation assay in MCF-7 cells. Scale bars $=50 \mu m$. (D) Quantitative measurement of EdU positive cells showed in C. (E) TUNEL assay in MCF-7 cells. Scale bars $=50 \mu \mathrm{m}$. (F) Quantitative measurement of TUNEL positive cells showed in E. Results are representative of at least 3 separate experiments and expressed as mean \pm SE. 
A

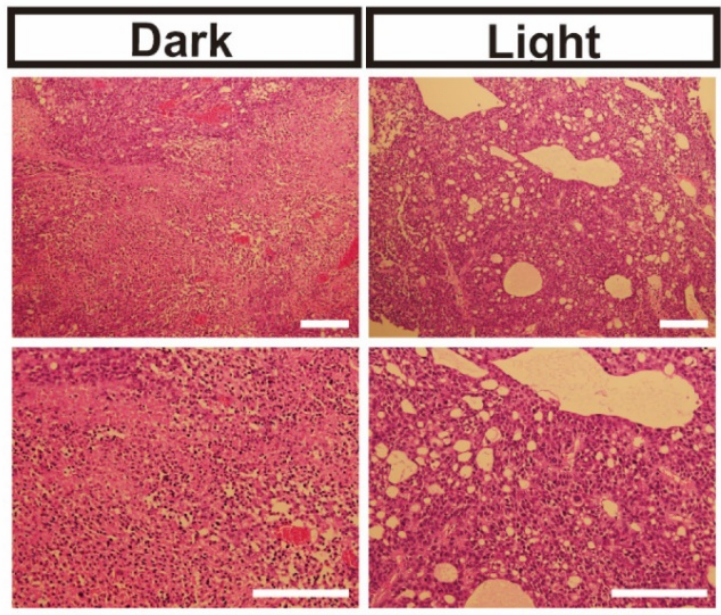

B

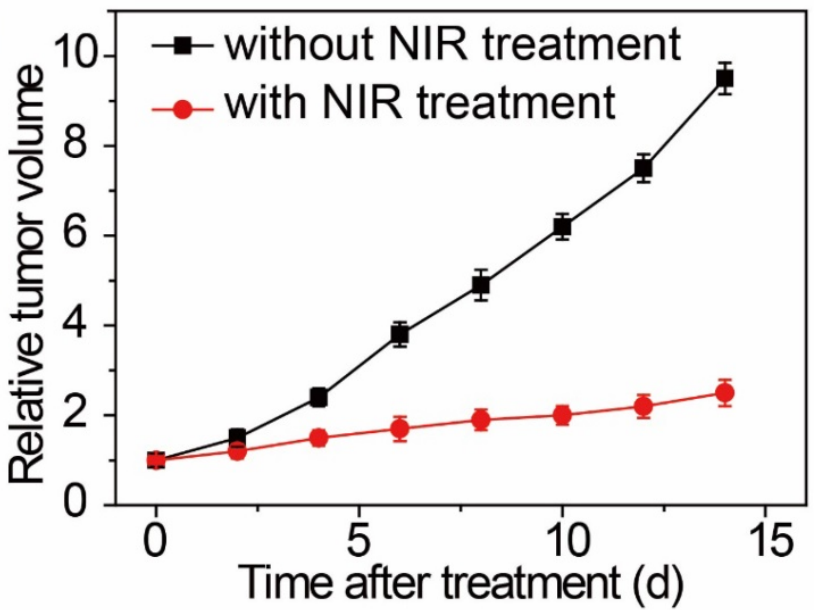

Figure 6. (A) Histological observation of the tumor tissues without and with NIR treatment hypodermic injection of the gold nanoparticle bouquet after 7 days. (B) Change of tumor volume with or without NIR treatment for 2 weeks. Scale bars: $200 \mu \mathrm{m}$.

\section{In vivo Studies}

On the basis of former cell NIR studies, we further established a binary system to monitor the effects of NIR therapeutic in living mice. Feminine pathogen free (5-6 weeks) BALB/c nude mice were used as tumor models. Under isoflurane anesthesia, MCF-7 cells in the logarithmic growth stage were injected subcutaneously into the flanks of the nude mice. Then, the AuNP60/APBA-AuNP13/MN nanoparticle bouquet solution was hypodermically injected into the tumor-bearing mice. The nanoparticle bouquet could efficiently arrival the tumor region, mice with the MCF-7 tumor were treated with a NIR laser (power of $0.5 \mathrm{~W} \mathrm{~cm}^{-2}$ ) irradiation for $30 \mathrm{~min}$ to treatment, while mice without NIR treatment were served for control. As shown in Figure 6, after 7 days of NIR treatment, the tumor status was monitored. Histological examination was carried out to observe the tumor tissues. The tumor tissue sections were clearly necrotic, while the control group showed no apparent cell destruction (Figure 6B). The results show that the proposed system NIR therapy has an obvious therapeutic effect on tumor tissue.

\section{Conclusion}

In this work, we designed the AuNP60/APBAAuNP13/MN nanoparticle bouquet to research the p53 in cells signal conductive pathway by photothermal therapy. The developed plasmonic AuNP60/APBA and AuNP13/MN sample can be easily prepared and had good biocompatibility. The AuNP60/APBA-wrapped AuNP13/MN readily entered living cells to do photothermal therapy.
Evidently, the color changes are caused by the conjugation of AuNP60/APBA and AuNP13/MN, the formation of AuNP60/APBA-AuNP13/MN nanoparticle bouquet was observed, the changes of the p53 level in cells were studied through NIR irradiation. Thus, results showed that the intracellular WTp53 content increases while the MTp53 content decreases. The method is applicable to detect the changes of p53 level in cells during AuNP60/APBAAuNP13/MN nanoparticle bouquet photothermal therapy, which is helpful to clarify the role of p53 in cell biological events. Thus, this capability demonstrates the effectiveness of the method for performing intracellular p53 analysis to target cancer therapy.

\section{Supplementary Material}

Supplementary figures.

http://www.ntno.org/v04p0201s1.pdf

\section{Acknowledgements}

We acknowledge Professor Yi-Tao Long's great help for establishing the dark-filed instrument system and providing the data analysis software. This research was supported by the National Natural Science Foundation of China (21904068, 81922041, 81570378 and 81772020), the Science and Technology of Jiangsu Province China (BK20170048), Jiangsu university natural science research program (19KJB150014), and Jiangsu Specially Appointed Professor program, Introduction of talent research start fund of Nanjing Medical University (NMUR2019007). 


\section{Competing Interests}

The authors have declared that no competing interest exists.

\section{References}

1. Jiang Y, Shi M- L, Liu Y, Wan S, Cui C, Zhang L- Q, Tan W-H. Aptamer/ AuNP Biosensor for Colorimetric Profiling of Exosomal Proteins. Angew. Chem. Int. Ed. 2017, 56(39): 11916-11920. doi: 10.1002/anie.201703807.

2. Qi G-H, Zhang Y, Xu S-P, Li C-P, Wang D-D, Li H-J, and Jin Y-D. Nucleus and mitochondria targeting Theranostic Plasmonic Surface-Enhanced Raman Spectroscopy Nanoprobes as a Means for Revealing Molecular Stress Response Differences in hyperthermia cell death between cancerous and normal cells. Anal. Chem. 2018, 90(22):13356-13364. doi.org/10.1021/acs.analchem.8b03034.

3. Huang P, Lin J, Li W-W, Rong P-F, Wang Z, Wang S-J, Wang X-P, Sun X-L, Aronova M, Niu G, Leapman R- D., Nie Z-H, Chen X-Y. Biodegradable gold nanovesicles with an ultrastrong plasmonic coupling effect for photoacoustic imaging and photothermal. Angew. Chem. Int. Ed. 2013, 52(52):13958-13964. doi: 10.1002 /anie.201308986.

4. Aioub M and El-Sayed M- A. A Real-Time surface enhanced Raman spectroscopy study of plasmonic photothermal cell death using targeted gold nanoparticles. J. Am. Chem. Soc. 2016, 138(4): 1258-1264. doi: 10.1021/jacs.5b10997.

5. Cheng X-J, Sun R, Yin L, Chai Z-F, Shi H-B, and Gao M-Y. Light-triggered assermbly of gold nanoparticles for photothermal therapy and photoacoustic imaging of tumors in vivo. Adv. Mater. 2017, 29(6): 1604894-1604900. doi: 10.1002/adma.201604894.

6. Lopez-Marzo A-M, Hoyos-de-la-Torre R, Baldrich E. NaNO3/ $\mathrm{NaCl}$ oxidant and Polyethylene glycol (PEG) capped gold nanoparticles (AuNPs) as a novel green route for AuNPs detection in electrochemical biosensors. Anal. Chem. 2018, 90 (6):4010-4018. doi: 10.1021/acs.analchem.7b05150.

7. Kim J, Oh S- Y, Shukla S, Hong S- B, Heo N-S, Bajpai V- K, Chun H- S, Jo C- H, Choi B- G, Huh Y- S, Han Y- K. Heteroassembled gold nanoparticles with sandwich-immunoassay LSPR chip format for rapid and sensitive detection of hepatitis B virus surface antigen (HBsAg). Biosens \& Bioelectron. 2018, 107:118-122. doi: 10.1016/j.bios.2018.02.019.

8. Yu R-J, Ying Y- L, Gao R, Long Y- T. Confined Nanopipette Sensing: From Single Molecules, Single Nanoparticles to Single Cells. Angew. Chem. Int. Ed. 2019, 58(12):3706-3714. doi: 10.1002/anie.201803229.

9. Aldewachi $\mathrm{H}$, Chalati $\mathrm{T}$, Woodroofe $\mathrm{M}-\mathrm{N}$, Bricklebank $\mathrm{N}$, Sharrack B, Gardiner P. Gold Nanoparticle-Based Colorimetric Biosensors. Nanoscale 2018, 10(1): 18-33. doi.org/10.1039/C7NR06367A.

10. Qian R- C, Cao Y, Long Y- T. Dual-Targeting Nanovesicles for In situ Intracellular Imaging of and Discrimination between Wild-type and Mutant p53. Angew. Chem. Int. Ed. 2015, 55(2):719-723. doi: 10.1002/anie.201510142.

11. Jing C, Gu Z, Ying Y- L, Li D- W, Zhang L, Long Y- T. Chrominance to Dimension: A Real-Time Method for Measuring the Size of Single Gold Nanoparticles. Anal. Chem. 2012, 84 (10):4284-4291. doi: 10.1021/ac203118g.

12. Kim J, Jo C, Lim W, Jung S, Lee Y- M, Lim J, Lee H, Lee J, Kim W- J. Programmed Nanoparticle-Loaded Nanoparticles for Deep-Penetrating 3D Cancer Therapy. Adv. Mater. 2018, 30(29):1707557-1707565. doi: 10.1002/adma.201707557.

13. Wang I, Zhang $\mathrm{Y}$, Jin $\mathrm{N}$, Mao C- B, Yang $\mathrm{M}-\mathrm{Y}$. Protein-Induced Gold Nanoparticle Assembly for Improving the Photothermal Effect in Cancer Therapy. ACS Appl. Mater. Interfaces 2019, 11 (12):11136-11143. doi: 10.1021/acsami.8b21488.

14. Jin R-H, Liu Z- N, Bai Y- K, Zhou Y- S, Gooding J- J, Chen X. Core-Satellite Mesoporous Silica-Gold Nanotheranostics for Biological Stimuli Triggered Multimodal Cancer Therapy. Adv. Funct. Mater. 2018, 28(31): 1801961-1801969. doi.org/10.1002/adfm.201801961.

15. Choi C. K. K, Chiu Y. T. E, Zhuo X. L, Liu Y, Pak C. Y, Liu X. D, Tse Y-L. S, Wang J. F. and Choi C. H. J. Dopamine-mediated assembly of citrate-capped plasmonic nanoparticles into stable core-shell nanoworms for intracellular applications. ACS Nano 2019, 13(5):5864-5884. doi: 10.1021/acsnano.9b01591.

16. Belhout S.A, Baptista F.R, Devereux S.J, Parker A.W, Ward A. D and Quinn S.J. Preparation of polymer gold nanoparticle composite with tunable plasmon coupling and their application as SERS substrates. Nanoscale, 2019, 11(42): 19884-19894. doi.org/10.1039/C9NR05014K.

17. Shen N, Yan F, Pang J- X, Gao Z, AI-Kali A, Haynes C- L, Litzow M- R, Liu S- J. HDL-AuNPs-BMS nanoparticle conjugates as molecularly targeted therapy for leukemia. ACS Appl. Mater. Interfaces 2018, 10(17):14454-14462. doi: 10.1021/acsami.8b01696.

18. Tornesello M- L, Annunziata C, Tornesello A- L, Buonaguro L, Buonaguro FM. Human Oncoviruses and p53 Tumor Suppressor Pathway Deregulation at the Origin of Human Cancers. Cancers 2018, 10(7):3892-3901. doi: $10.3390 /$ cancers 10070213.

19. He F, Borcherds W, Song T- J, Wei X, Das M, Chen L- H, Daughdrill G- W, Chen J- D. Interaction between p53 N terminus and core domain regulates specific and nonspecific DNA binding. PNAS 2019, 116(18): 8859-8868. doi: 10.1073 / pnas.1903077116.
20. Aggarwal M, Saxena R, Asif N, Sinclair E, Tan J, Cruz I, Berry D, Kallakury B, Pham Q, Wang T T-Y, Chung F- L. p53 mutant-type in human prostate cancer cells determines the sensitivity to phenethyl isothiocyanate induced growth inhibition. J Exp Clin Canc Res. 2019; 38(1):307-324. doi: 10.1186/s13046019-1267-z.

21. Greenblatt M- S, Bennett W- P, Hollstein M, Harris C- C. Mutations in the p53 Tumor Suppressor Gene: Clues to Cancer Etiology and Molecular Pathogenesist. Cancer Res. 1994, 54(18):4855-4878.

22. Maritschnegg E, Heinzl N, Wilson S, Deycmar S, Niebuhr M, Klameth L, Holzer B, Koziel K, Concin N. Zeillinger R. Polymer-ligand-based ELISA for robust, high throughput, quantitative detection of p53 aggregates Anal. Chem. 2018, 90(20): 13273-13279. doi: 10.1021/acs.analchem.8b02373.

23. Cole A- J, Dwight T, Gill A- J, Dickson K- A, Zhu Y, Clark-son A, Gard G- B, Maidens J, Valmadre S, Clifton-Bligh R, Marsh D- J. Assessing mutant p53 in primary high-grade serous ovarian cancer using immunohistochemistry and massively parallel sequencing. Scientific Reports. 2016; 6:26191-26203. doi: 10.1038 /srep26191.

24. Ream J- A, Lewis L- K, Lewis K- A. Rapid agarose gel electrophoretic mobility shift assay for quantitating protein: RNA interactions. Anal. Biochem. 2016; 511:36-41. doi: 10.1016/j.ab.2016.07.027.

25. Oian R- C, Cao Y, Zhao L- J, Gu Z, Long Y-T. A Two-Stage Dissociation System for Multilayer Imaging of Cancer Biomarker-Synergic Networks in Single Cells. Angew. Chem. Int. Ed. 2017, 56 (17):4802-4805. doi: 10.1002/anie.201702415.

26. Deshpande N, Ramesh A, Nandi D, Nguyen A, Brouillard A, Kulkarni A. Supramolecular polysaccharide nanotheranostics that inhibit cancer cells growth and monitor targeted therapy response. Nanotheranostics 2020, 4(3):156-172. doi: 10.7150/ntno.44703.

27. Cheung C C. L., Ma G- L, Karatasos K, Seitsonen J, Ruokolainen J, Koffi C -R, H Hassan A.F.M, Al-Jama W T. Liposome-templated indocyanine green Jaggregates for in vivo near infrared imaging and stable photothermal heating. Nanotheranostics 2020, 4(2):91-106. doi: 10.7150/ntno.41737. eCollection 2020.

28. Cao Y, Xie T, Qian R- C, Long Y-T. Plasmon Resonance Energy Transfer: Coupling between Chromophore Molecules and Metallic Nanoparticles. Small 2017, 13(2): 1318-1322. doi: 10.1002/smll.201601955

29. Han E, Ding L, Ju H- X. Highly Sensitive Fluorescent Analysis of Dynamic Glycan Expression on Living Cells Using Glyconanoparticles and Functionalized Quantum Dots. Anal. Chem. 2011, 83(18): 7006-7012. doi: $10.1021 /$ ac201488x

30. Liu Y, Ai K, Liu J, Deng M, He Y, Lu L. Dopamine-Melanin Colloidal Nanospheres: An Efficient Near-Infrared Photothermal Therapeutic Agent for In vivo Cancer Therapy. Adv. Mater. 2013, 25(9): 1353-1359. doi: 10.1002/adma.201204683. 